\title{
Infinite symmetric nuclear matter phase diagram from low energy compound nucleus data.
}

\author{
Luciano Moretto*, James B Elliott ${ }^{\dagger}$, Peter T Lake, Larry Phair \\ $L B N L$ and UCB Berkeley \\ E-mail: lgmoretto@lbl.gov
}

\begin{abstract}
The inherent mesoscopic nature of nuclei and the Coulomb interaction make it difficult to cast systems interacting through the strong force into thermodynamic terms. Our task is to extract the phase diagram of the theoretical infinite symmetrical uncharged nuclear matter from experiments of nuclear collisions where the systems are neither infinite, symmetrical, nor uncharged. A framework of how to relate isotopic yields of such experiments to coexistence densities and pressures is set in place by use of Fisher's droplet model. The theory is applied to the model systems of the Ising model and a system of particles interacting via the Lennard-Jones potential. The specific details inherent to nuclear reactions are considered. These include finite size effects, the Coulomb repulsion, and the lack of a physical vapor in contact with a decaying system. Experimental data of compound nucleus experiments are studied within the theory presented, which is also shown to extend to higher energy reactions. With all these considerations, the phase diagram of nuclear matter is extracted.
\end{abstract}

XLIX International Winter Meeting on Nuclear Physics

January 24-28, 2011

BORMIO, Italy

\footnotetext{
†Current Address:

Lawrence Livermore National Laboratory, 94551 Livermore, CA, USA

* Speaker.
} 


\section{Introduction}

Microscopically, the nature of the forces holding a nucleus together being repulsive at short distances with a diffuse attractive potential well at larger distances is analogous to the forces holding a drop of water together. It is then natural to expect that the nuclear system will exhibit a liquid-vapor phase transition just as their atomic and molecular brethren do. Macroscopically, there is no way to study a bulk sample of nuclear matter to characterize it in the same fashion as these other analogous systems. As a result, the exact nature of the nuclear matter phase diagram is unknown.

The experimental data that is accessible for the nuclear system comes from nuclear reactions. These reactions can range from lower energy that are characterized in terms of compound nuclear decays to higher energy where it is more appropriate to talk in terms of multifragmentation. These reactions range from collision energies of $6 \mathrm{AMeV}$ to $1 \mathrm{AGeV}$.

Many signals in such experiments have been used to claim that there is indeed a phase transition in nuclear matter. These signals have included studying bimodality and the caloric curve. May these signals actually be due to the phase transition or not is irrelevant to the fact that none of them have given rise to a phase diagram of nuclear matter. We have chosen to study cluster yields as a means to study the phase transition in hopes of ultimately creating a phase diagram [1].

In previous studies, the concepts of thermalization and reducibility have been introduced to describe the cluster yields of nuclear reactions [2]. Even though the actual physics behind these nuclear collisions are complex, only a small number of parameters are needed to characterize the process. The process of thermalization is how the energy of the collision is distributed over many
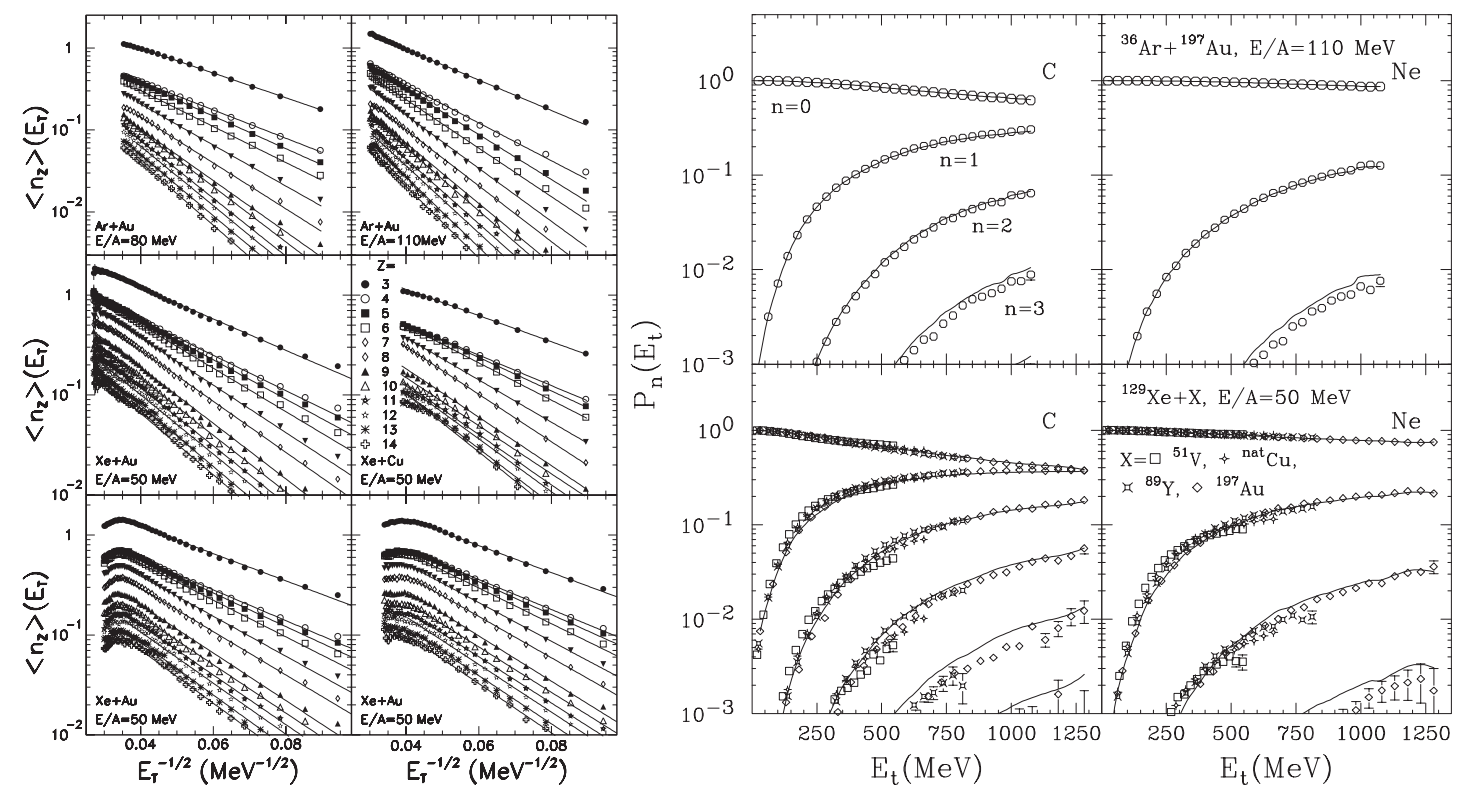

Figure 1: Left: The average yield as a function of the square root of the transverse energy. The symbols show experimental data points while the solid lines show fits to the data using a Boltzmann form. Right: The excitation function for carbon (left) and neon (right) emission. The symbols show experimental data points and the solid curves are Poisson fits. See reference [2] for further details. 
degrees of freedom of the system whereby generating a temperature. An Arrhenius plot, like those shown in figure 1 , shows that the emission rates of clusters as a function of inverse temperature creates a linear plot as expected for a thermal system. Furthermore, reducibility is the idea that the number of large fragments being emitted from the hot source can be described in terms of a one fragment probability. Figure 1 also shows how the probabilities of multiple large fragment emissions can be parametrized by the one fragment probability. Together, thermalization and reducibility show that the clusters emitted from such reactions are thermal and stochastic.

The task in front of us is to connect these thermal cluster yields, being microscopic in nature, to the macroscopic characterization of the system in terms of a phase diagram. Using the physical cluster model and Fisher's droplet model [3,4], we study the clusters found in the Ising model and a system interacting through a Lennard-Jones potential. With the cluster concentrations from simulations, the phase diagrams of the systems can be extracted.

With a connection between the cluster concentrations and the thermodynamic variables of a system, the peculiarities of the nuclear system need to be considered. Since the system is never studied as a vapor in direct contact with its coexisting liquid, three details need to be considered:

1. The finite nature of the liquid drop as opposed to a bulk sample.

2. The presence of the Coulomb repulsion.

3. The fact that the liquid evaporates into a vacuum and not into its coexisting vapor.

Armed with these considerations, we turn our attention to the data from experiments and see that the expected trends are observed. From this data, we can achieve our goal of constructing the phase diagram of nuclear matter.

\section{Cluster concentrations of coexisting systems}

\subsection{Physical cluster model}

Cluster models were originally developed to account for the non-ideal nature of real fluids. The purpose of the physical cluster model is to treat the vapor as an ideal gas of clusters. As a result, the following two relations are established for the density, $\rho$, and pressure, $p$, of the system as a function of the cluster concentrations of $\operatorname{size} A, n_{A}$ :

$$
\begin{aligned}
& \rho=\sum_{A} A n_{A}, \\
& p=T \sum_{A} n_{A} .
\end{aligned}
$$

The first equation is true for any partitioning of the system into clusters. The second equation is not trivially true for any partition, and can be seen as the sum over partial pressures of an ideal gas of each cluster size.

These equations also hold the key in relating the microscopic concept of clusters to the thermodynamic variables of pressure and density. The question that remains is to find a suitable theory to describe the cluster concentrations, $n_{A}$. 


\subsection{Fisher's droplet model}

Fisher's droplet model is a phenomenological approach to enumerating the cluster concentrations [3, 4]. Generically, the concentration of a cluster should be equal to a Boltzmann factor, incorporating the change of free energy, $\Delta F_{A}$, to take a cluster of a given size from the liquid into the vapor phase:

$$
n_{A}=\exp \left[-\Delta F_{A} / T\right]=\exp \left[-\left(\Delta E_{A}-T \Delta S_{A}\right) / T\right] .
$$

The change in energy, $\Delta E_{A}$, is seen to be the energy associated with creating the surface of a cluster:

$$
\Delta E_{A}=c_{0} A^{\sigma}
$$

The relation between this equation and the liquid drop expansion are evident. The $c_{0}$ is the surface energy coefficient, and $\sigma$ would be $2 / 3$ for a spherical drop. Since the smaller clusters are not spherical, strictly speaking, the value of $\sigma$ is allowed to change from this ideal value.

Fisher's contribution to evaluating the cluster concentrations comes in writing the change in entropy, $\Delta S_{A}$, as follows:

$$
\Delta S_{A}=\log \left[q_{0}\right]-\tau \log [A]+\frac{c_{0} A^{\sigma}}{T_{c}} .
$$

To better see how this describes the cluster entropies phenomenologically, consider the resulting cluster concentrations using the above two equations:

$$
n_{A}=q_{0} A^{-\tau} \exp \left[-c_{0} A^{\sigma}\left(\frac{1}{T}-\frac{1}{T_{c}}\right)\right] .
$$

For a system at the critical point, one expects the cluster concentrations to follow a power law, which is captured in the above equation. The parameter $\tau$ is seen to be the critical exponent to describe that power law. The value $q_{0}$ is the proportionality constant.

The aspect of the above equation which we take advantage of is that $T_{c}$ is a parameter. Fitting the cluster concentrations to Fisher's formula gives the critical temperature of the system, and the phase diagrams can be produced by using equations 2.1 and 2.2.

\section{Clusters in model systems}

To test the validity of Fisher's equation for cluster concentrations, we chose two model systems to study. The phase diagrams of these systems are well understood, and our approach is to see how well the phase diagrams can be reproduced via the cluster concentrations. The two systems are the Ising model and the Lennard-Jones model.

\subsection{The Ising model}

The Ising model is a system of spins confined to a grid which interact with nearest neighbors. The energy of a given lattice configuration is:

$$
E=-J \sum_{\langle i, j\rangle} s_{i} s_{j}-H \sum_{i} s_{i} .
$$


The first sum is over all nearest neighbors, and $J$ is the interaction energy between two spins. The second sum represents the energy of the spins interacting with an external magnetic field with strength $H$.

Even though this system models the ferromagnetic phase transition, it is seen to be related to the lattice gas model. Specifically, in no external field, $H=0$, the system is isomorphic to the lattice gas at coexistence [5,6]. Thus the phase transition in the Ising model is analogous to the liquid-vapor phase transition.

For this study, calculations were performed using the Swendson-Wang algorithm [7], a standard Monte Carlo technique, on a cubic lattice with a linear dimension of 50 cells. Periodic boundary conditions were used to minimize the finite size effects. Since we are interested here in the nature of the system at coexistence, no external field is used. The clusters were defined by using the Coniglio-Klein algorithm, dividing the geometric clusters into smaller partitions [8].

\subsection{The Lennard-Jones model}

The Lennard-Jones model is an off-lattice system where the particles interact with a pairwise potential of the form:

$$
V_{L J}(r)=-4 \varepsilon\left[\left(\frac{r_{L J}}{r}\right)^{-6}-\left(\frac{r_{L J}}{r}\right)^{-12}\right] .
$$

Here, $r$ is the distance between the two particles and $\varepsilon$ and $r_{L J}$ are the characteristic energy and distance of the system, respectively.

To study the system at coexistence the Gibbs ensemble method was employed [9]. A system of 4800 particles was studied. The volume of each phase has a linear dimension of about $20 r_{L J}$. Even though both the liquid and vapor are generated from this process, we are concerned here with only the vapor phase. The clusters were defined using Hill's procedure [10]. Random momenta are assigned to each particle since the Monte Carlo simulation does not produce them.
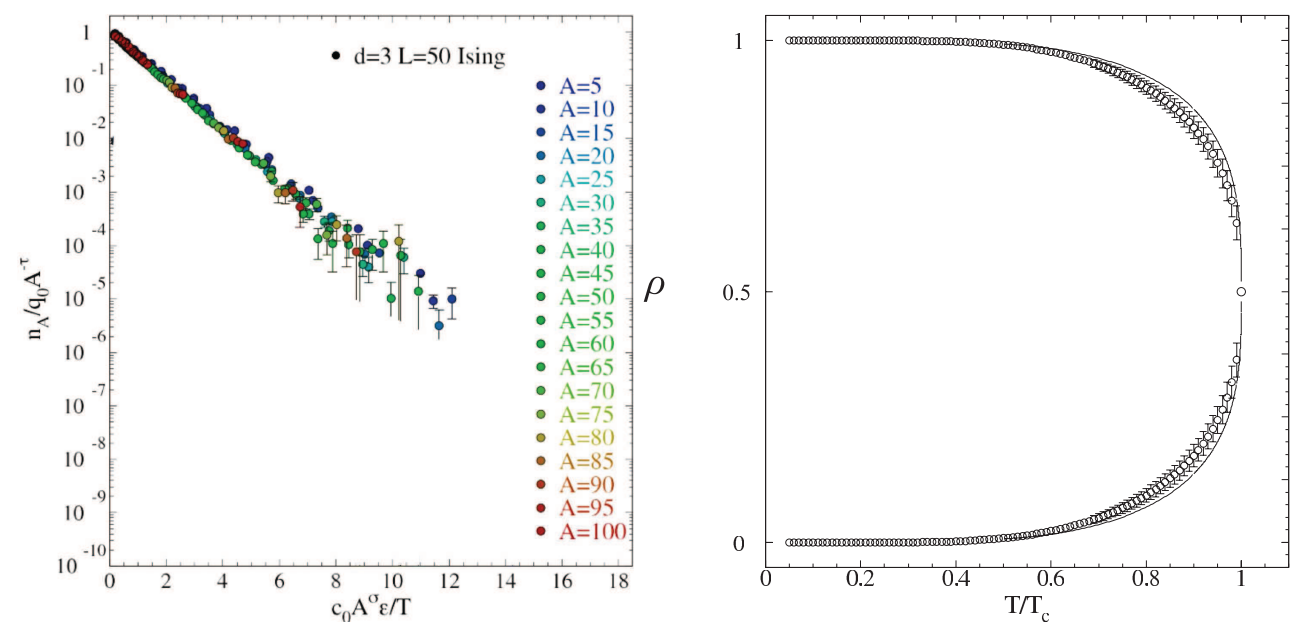

Figure 2: Left: Fisher scaling of the cluster concentrations in the Ising model using equation 3.3. Right: The phase diagram of the Ising model. The symbols are the extracted values from Fisher's theory and the line is the actual density of the system. [11, 15] 

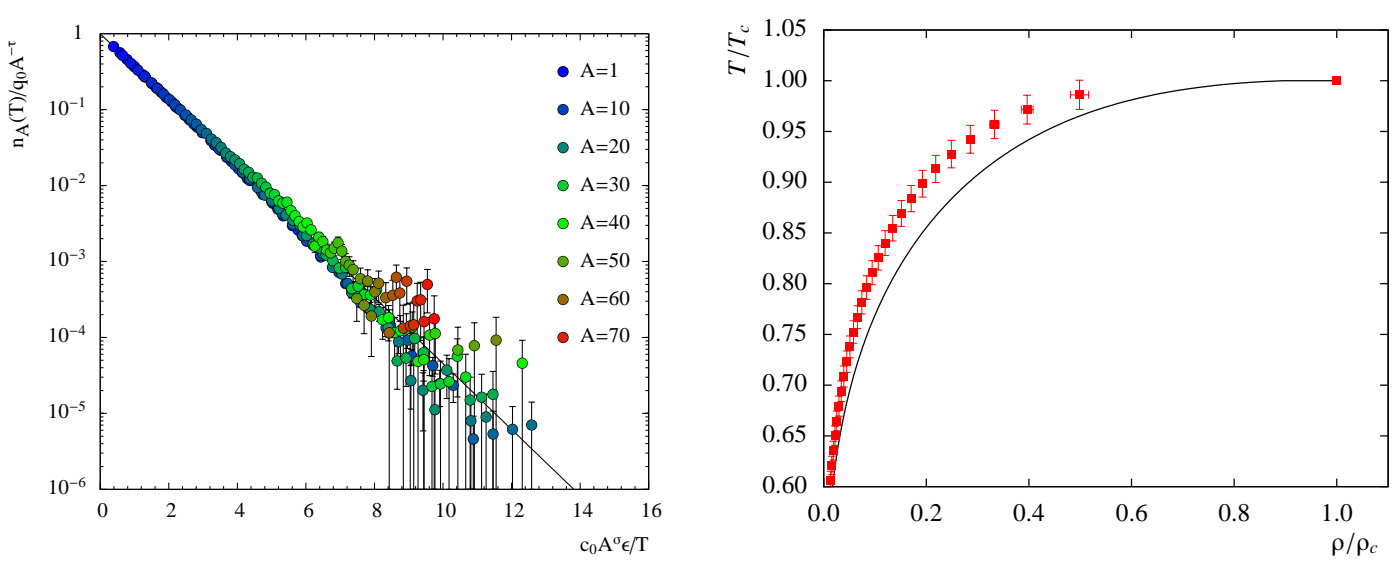

Figure 3: Left: Fisher scaling of the cluster concentrations in the Lennard-Jones model using equation 3.3. Right: The phase diagram of the Lennard-Jones model. The symbols are the extracted values from Fisher's theory and the line is the actual density of the system. [14]

\begin{tabular}{ccccc}
\hline & \multicolumn{2}{c}{ Ising Model } & \multicolumn{2}{c}{ Lennard-Jones Model } \\
& theoretical values & this work & theoretical values & this work \\
$T_{c}$ & $4.51152 \pm 0.00004[12]$ & $4.52 \pm 0.01$ & $1.3120 \pm 0.0007[13]$ & $1.368 \pm 0.002$ \\
$\sigma$ & $0.63946 \pm 0.0008$ & $0.732 \pm 0.008$ & $0.63946 \pm 0.0008$ & $0.744 \pm 0.002$ \\
$\tau$ & $2.209 \pm 0.006$ & $2.30 \pm 0.08$ & $2.209 \pm 0.006$ & $2.199 \pm 0.005$ \\
\hline
\end{tabular}

Table 1: Extracted parameters from the cluster scaling compared to the accepted theoretical values.

\subsection{Fisher scaling in model systems}

Once the cluster concentrations are calculated in the above systems, they can be fit to equation 2.6. A convenient way of plotting the data is by making a Fisher plot. Upon rearrangement of equation 2.6, one finds:

$$
\frac{n_{A}(T)}{q_{0} A^{-\tau}}=\exp \left[-c_{0} A^{\sigma}\left(\frac{1}{T}-\frac{1}{T_{c}}\right)\right] .
$$

In plotting the logarithm of the left hand side as a function of the argument of the exponential, all the data collapses onto one line irrespective of temperature and cluster number.

Figures 2 and 3 show the Fisher plots generated for both the Ising and Lennard-Jones models, respectively. Both systems display Fisher scaling. Furthermore, the extracted parameters of the fits replicate the theoretical values of the systems as seen in table 1 .

Also shown in figures 2 and 3 are the extracted density-temperature phase diagrams using the sums in equations 2.1 and 2.2. Even though these results are not close to the precision of more typical procedures, it is evident that the cluster scaling does indeed replicate the diagrams.

\section{Nature of the cluster yields from nuclear reactions}

Now that we have shown that cluster scaling can be used to create a phase diagram, we turn our attention to the nuclear system. Nuclear reactions do not result in a system at coexistence. 
Rather, it is a charged finite system decaying in a vacuum. To relate the cluster yields found in experimental data to those that would be found in a system at coexistence, three considerations must be made. First, the finite nature of the liquid drop affects the yields relative to the infinite system. The presence of the Coulomb repulsion must be accounted for, since we want to consider only the phase diagram arising from the nuclear matter interacting via the strong force. Lastly, the relation between the system decaying into a vacuum is compared to the system in equilibrium with its coexisting vapor. Once these three topics are addressed, the experimental data can be tamed.

\subsection{Finite size corrections}

The mesoscopic nature of nuclei make the study of finite size effects imperative. For the properties of ground state nuclei, these finite size effects are embodied in the liquid drop expansion [16]. In the liquid drop expansion, the binding energy is written as a term linear with size and a term that goes as the size to the two-thirds power. The former is seen to be a property of bulk nuclear matter, whereas the latter represents the finiteness of the nucleus and the presence of a surface.

To see how the finite size affects the cluster yields, return to equation 2.3 from the theory of cluster concentrations:

$$
n_{A}=\exp \left[-\Delta F_{A} / T\right]=\exp \left[-\left(\Delta E_{A}-T \Delta S_{A}\right) / T\right] .
$$

The $\Delta E_{A}$ and $\Delta S_{A}$ were seen to be the change of the respective variable as a cluster was removed from the liquid phase and placed in the vapor. In the case of a bulk sample of liquid, the properties of the liquid are unchanged, and the change of energy and entropy are just the creation of the cluster's surface. In a finite system with $A_{0}$ particles in a liquid drop, removing a cluster changes the size of the drop and hence changes the surface of the drop. The resulting smaller liquid drop, the "complement", must be taken into account [17]. The change in energy is therefore:

$$
\Delta E_{A}=c_{0}\left\{A^{\sigma}+\left(A_{0}-A\right)^{\sigma}-A_{0}^{\sigma}\right\} .
$$

This is the negative Q-value of the reaction, just as would be expected from a nuclear decay. The change in entropy is also affected by the presence of the liquid drop:

$$
\Delta S_{A}=-\tau \log \left[\frac{A\left(A_{0}-A\right)}{A_{0}}\right]+\frac{c_{0}}{T_{c}}\left\{A^{\sigma}+\left(A_{0}-A\right)^{\sigma}-A_{0}^{\sigma}\right\} .
$$

Collecting the terms together into equation 4.1, the cluster concentrations are re-evaluated for the case of a vapor in coexistence with a liquid drop:

$$
n_{A}=\left(\frac{A\left(A_{0}-A\right)}{A_{0}}\right)^{-\tau} \exp \left[-c_{0}\left\{A^{\sigma}+\left(A_{0}-A\right)^{\sigma}-A_{0}^{\sigma}\right\}\left(\frac{1}{T}-\frac{1}{T_{c}}\right)\right] .
$$

In comparison to the bulk system, the vapor is populated with more clusters:

$$
n_{A}\left(T, A_{0}\right)=n_{A}^{\infty}(T) \exp \left(A \Delta \mu_{\mathrm{fs}} / T\right) .
$$

Here, $n_{A}^{\infty}$ is the cluster concentrations in the bulk, and the presence of the liquid drop is to shift the chemical potential of the system relative to the bulk system by $\Delta \mu_{\mathrm{fs}}$ :

$$
\Delta \mu_{\mathrm{fs}}=-\frac{1}{A}\left(c_{0} \varepsilon\left[\left(A_{0}-A\right)^{\sigma}-A_{0}^{\sigma}\right]-T \tau \log \left[\frac{A_{0}-A}{A_{0}}\right]\right) .
$$


As would be expected, $\Delta \mu_{\mathrm{fs}}$ tends to zero when the liquid drop size becomes large; as the liquid becomes larger the finite size effects become smaller. These equations are directly related to the Gibbs-Thomson effect of increased vapor pressure in the presence of a liquid drop [18].

\subsection{Finite size correction in model systems}

To study the complement correction, the two model systems that were used previously to study Fisher scaling are used again. Unlike the grandcanonical calculations done in the case of studying the bulk coexistence, particle number must be conserved to stabilize a liquid drop.

For the Ising model, a canonical Monte Carlo simulation is performed on a lattice with a linear dimension of $L=25$ cells [17]. A relatively small number of the cells are set spin up, ranging from

$A_{0}^{d}=117$ to $A_{0}^{d}=468$. Coexistence is studied up to a certain temperature at which a liquid drop is no longer stable and the system becomes one homogeneous phase. Since the liquid drop is itself not part of the vapor, its volume is not included in measuring the cluster concentrations, which are otherwise measured in the same matter as before.

For the Lennard-Jones model, a microcanonical molecular dynamics simulation is performed in a spherical container with elastic walls. A constant $A_{0}^{d}=600$ is used, and various droplet sizetemperature combinations are obtained by varying the energy and container size. As with the Ising model, the volume of the liquid drop is identified and excluded from evaluating the cluster concentrations.

The results of these two sets of simulations are presented in figure 4. The top two figures show the Fisher scaling for the bulk system, as discussed in the previous section. Using the parameters from the bulk systems, the cluster concentrations from the liquid drop simulations are plotted in the middle panel. It is clear the Fisher scaling is not the same, and there is an excess of clusters relative to the bulk. The bottom panel then shows the clusters scaled using the complement corrected Fisher scaling from equation 4.4. The cluster concentrations are seen to be described correctly by the complement correction.

It should be noted that the collapse of the liquid drop data uses the parameters from the bulk system. This shows how the two systems are connected, and that even though the idea of a critical temperature is ambiguous for the case of a finite system, the critical temperature of the infinite system determines the cluster scaling in the finite system. Furthermore, the reverse procedure can be performed, where the clusters from the finite system can be fit to the Fisher complement relation, regaining the parameters of the infinite system.

\subsection{Coulomb repulsion}

The presence of a $1 / r$ potential, such as the Coulomb repulsion, makes it impossible to describe a bulk system thermodynamically. This is the reason why we are considering uncharged nuclear matter. The question is how to handle the Coulomb force which is ever present in nuclei, and extract information of the system as if it is not there [19]. 

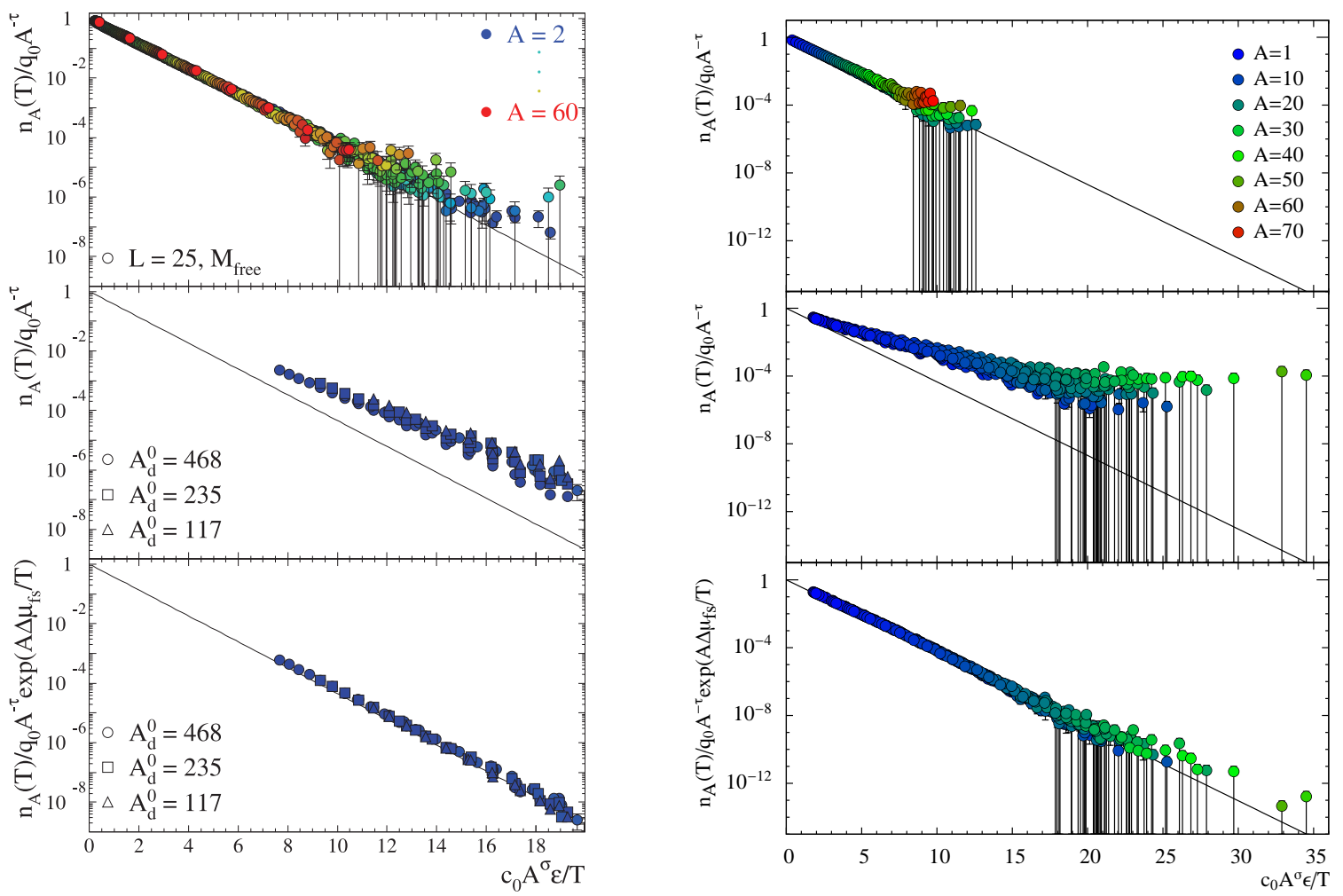

Figure 4: Top: Fisher scaling of the cluster concentrations for the infinite system using equation 2.6. Middle: Fisher scaling of the cluster concentrations for the system with a liquid drop using equation 2.6. Bottom: Fisher scaling of the cluster concentrations for the system with a liquid drop including the complement correction using equation 4.4. For all of the plots, the left correspond to the Ising model and the right to the Lennard-Jones model.

The Coulomb problem can be separated into three separate problems:

1. The liquid self energy.

2. The vapor self energy.

3. The liquid-vapor interaction energy.

The first problem is solved by use of the liquid drop expansion. The Coulomb term in the liquid drop expansion represents the loss of binding due to the liquid self energy. Since nuclei are finite in size in nuclear collisions, the Coulomb term stays finite and can easily be subtracted from the the total binding to leave behind the parts characteristic to the strong force.

The second problem is a problem for considering a gas of nucleons. With the Coulomb repulsion, the only stable configuration of the vapor would be a vapor of zero density. Since there is never a vapor in coexistence with the liquid drop in a nuclear collision, this problem becomes irrelevant. The assumption made is that a cluster emitted from the source immediately leaves and does not interact with any other outgoing cluster. In this respect, the lack of a physical vapor in contact with the liquid drop is not a hindrance, but makes this analysis possible.

In principle, the third problem is actually not a problem at all. To have coexistence between two phases, these phases do not need to be in contact. As long as the two systems are at the same temperature and chemical potential, coexistence is guaranteed. The presence of a surface between 


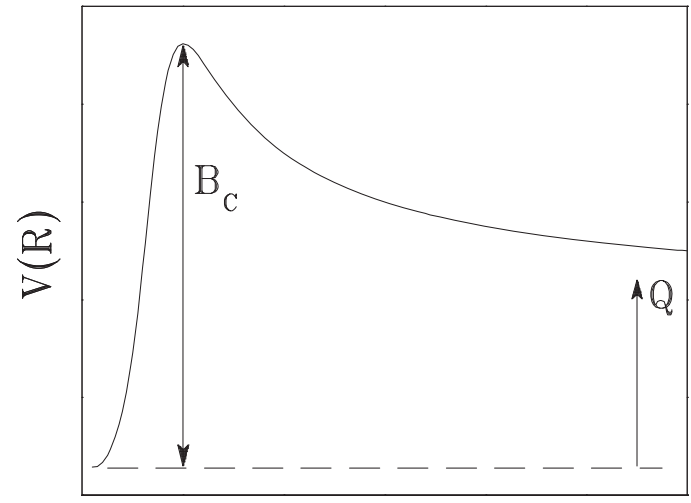

$\mathrm{R}$

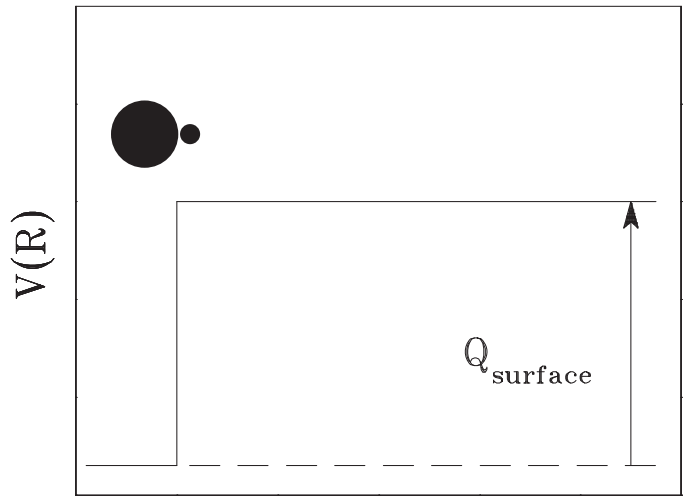

$\mathrm{R}$

Figure 5: Schematic potential energy curves for cluster emission with (left) and without (right) the Coulomb interaction. The only difference between the emission of the cluster in the two cases is the presence of an extra barrier when the Coulomb interaction is present.

the two is irrelevant to the thermodynamic properties of the two independent phases. As a result, there need not be any interaction energy between the two phases to properly describe coexistence.

In the hot source generated after a nuclear collision, the Coulomb interaction energy produces a barrier to cluster emission. This barrier does not change the final equilibrium of the system, as seen in the previous argument, but changes the emission rate. The evaporation of clusters can be cast in terms of transition state theory [20]. Consider the potential energy curves shown in figure 5. With no Coulomb, the energy of the system emitting at the surface of the source is the same as the energy of the two being separated at infinity. With Coulomb, this is no longer true, and the additional energy can be modeled as the interaction energy of two touching charged spheres. The rate of emission, $R$, is thus modeled by:

$$
R \propto \exp \left[-B_{s} / T\right]=\exp \left[-\left(\Delta E_{\text {surface }}-\Delta E_{\text {Coulomb }}\right) / T\right]
$$

where the $\Delta E$ terms represent the barrier created from the two different interactions. Hence, the rates are hindered by a Boltzmann factor of the Coulomb barrier. Dividing this factor from the observed rates yields the rate that would be expected for the system with no Coulomb interaction.

Even though the Coulomb interaction would make it impossible to define the thermodynamic variables of the nuclear system, the fact that we can only study finite systems makes it possible to account for the repulsion. In the case of decay from a hot source, the only effect of the Coulomb interaction is to create a barrier to emission. This hindrance to decay can be divided out of the yields observed, and what remains are the yields of an uncharged system.

\subsection{Emission into a vacuum}

Without a coexisting vapor in contact with the liquid, how can the decay rates of a liquid drop in vacuum be associated with the equilibrium concentrations [21]?

To see how this apparent contradiction is resolved, consider the two experimental detection schemes show in figure 6. Each case represents an enclosed system. The first is a system in 

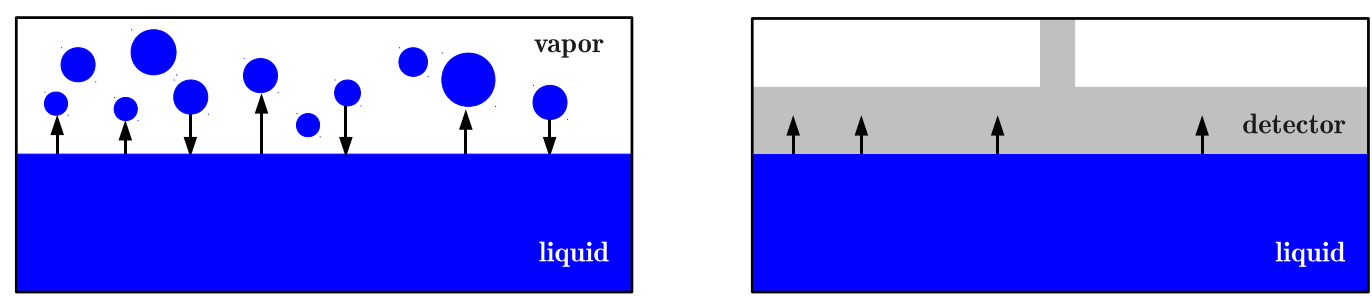

Figure 6: Schematic representation of the rate of particles crossing the liquid-vapor boundary for two different experimental set-ups. It is seen that the outward flux of particles from the liquid is identical with or without the presence of the vapor phase.

coexistence with the top wall of the container being a detector. The detector measures the rate at which particles hit the wall.

The position is irrelevant as long as the pressure and temperature are kept constant in the system. As a result, moving the detector all the way to the surface of the liquid results in the same measurement. Thus, the outward flux of particles from the liquid is equal to the flux of particles from the coexisting vapor hitting the wall.

For an ideal vapor, as the physical cluster model presumes, the rate of particles, $R_{A}$, impinging upon a wall is written as:

$$
R_{A}(T)=n_{A}(T)\left\langle v_{A}(T) 4 \sigma_{i n v}\left(v_{A}\right)\right\rangle .
$$

Here, $n_{A}$ is the equilibrium concentration, $v_{A}$ is the velocity of the particle, and $\sigma_{i n v}$ is the inverse cross section. The term $v_{A}$ is of order $\sqrt{T / A}$, and $\sigma_{i n v}$ is of order $A_{0}^{2 / 3}$, where $A_{0}$ is the mass number of the hot source.

Another difference between the equilibrated system and a system decaying into a vacuum is that the decaying system cools down as it evaporates particles. At first it would appear that the temperature of the system needs to be known for the whole history of the evaporation. To avoid this complication, only the intermediate mass fragments are considered, with charge of more than $Z=6$. These larger fragments have a large barrier for emission, not only due to the increase of surface, but more so because of the Coulomb barrier as discussed in the previous section. As a result, the larger fragments are considered to be emitted first, or not at all.

A complete physical picture of the evaporation process is thus made. After the collision, the system quickly equilibrates to a temperature. The resulting hot liquid drop loses its energy through particle emission. The large fragments are only emitted when the system has this large initial energy, and their rate of emission is directly related to the coexisting vapor concentrations through equation 4.8. The system then continues its evaporative cooling, but overwhelmingly emitting only the smaller mass fragments.

\section{Experimental fragment scaling}

With the above considerations of cluster scaling and the details of the nuclear system, we turn our attention to the fragment distributions from experimental measurements of nuclear reactions. Two types of experiments are used to create the phase diagrams of nuclear matter. 


\begin{tabular}{cc}
\hline Reaction & $T_{c}(\mathrm{MeV})$ \\
${ }^{58} \mathrm{Ni}+{ }^{12} \mathrm{C} \rightarrow{ }^{70} \mathrm{Se}$ & $18.4 \pm 0.3$ \\
${ }^{64} \mathrm{Ni}+{ }^{12} \mathrm{C} \rightarrow{ }^{76} \mathrm{Se}$ & $18.0 \pm 0.2$ \\
$1 \mathrm{AGeV}{ }^{84} \mathrm{Kr}+{ }^{12} \mathrm{C}$ & $17.5 \pm 0.2$ \\
$1 \mathrm{AGeV}{ }^{139} \mathrm{La}+{ }^{12} \mathrm{C}$ & $18.3 \pm 0.2$ \\
$1 \mathrm{AGeV}{ }^{197} \mathrm{Au}+{ }^{12} \mathrm{C}$ & $17.7 \pm 0.1$ \\
$1 \mathrm{GeV} / \mathrm{c} \pi+{ }^{197} \mathrm{Au}$ & $17.26 \pm 0.02$ \\
\hline
\end{tabular}

Table 2: Estimates of the critical temperature of nuclear matter from six different nuclear reactions.

One type of experiment gives rise to a standard "compound nucleus". These nuclear collisions are characterized as when the beam and target nuclei fuse into a single highly excited nucleus. The resulting compound nucleus is easily parametrized by mass number, excitation energy, and angular momentum through conservation laws [22].

The other experiments are loosely grouped into what is call "multifragmentation" [23, 24, 25, 26, 27, 28, 29, 30, 31, 32, 33]. These experiments are at much higher incident particle energies. As a result, a single compound nucleus is not formed. Rather, the collision breaks the two nuclei apart, leaving behind a smaller remnant which contains only a small amount of the incident beam energy. It is harder to characterize the remnant in the same terms as the compound nucleus, but in principle they decay in the same fashion. The remnant quickly equilibrates and cools by means of evaporating clusters. Similar studies have been performed on such experimental results, however the Coulomb force and finite size effects were not properly taken into account [24, 25, 34].

Regardless of the means of formation of the highly excited remnant and/or the compound nucleus, the decay thereafter is all considered in the same formalism. Each system is characterized in terms of a mass number, charge, excitation energy, and angular momentum. The excitation energy is transformed into a temperature via the Fermi gas approximation [36, 37]. The effects of angular momentum of the decaying source is also considered. The resulting cluster yields are then analyzed as described in the previous sections. Complete and in depth details of this analysis can be found in reference [35].

The data from six different reactions (listed in Table 2) and three different experiments were used and over 500 data points were fit with three (for the compound nuclear data sets) or four (for the multifragmentation data sets) free parameters per reaction (there were, on average, nearly 23 data points per free parameter). Charges from $6 \leq Z_{f} \leq 25$ and excitation energies of 1.08 $\mathrm{AMeV} \leq E_{r}^{*} \leq 4.75 \mathrm{AMeV}$ were used in the analysis. Table 2 shows the results for $T_{c}$ from all the experiments. These values agree with each other to within $3 \%$ and give an estimate of the critical temperature of bulk nuclear matter as $T_{c}=17.9 \pm 0.4 \mathrm{MeV}$. This value agrees well with theoretical predictions [38, 39, 40, 41, 42].

Plotting the scaled ratio of the yields of a given fragment $Y\left(Z_{r}, A_{r}\right)$ as a function of as $A^{\sigma} \varepsilon / T$ collapses the measured fragment yields for any $A_{f}$ and $E_{r}$ onto a single curve. This is shown in Figure 7. The curve is, for all intents and purposes, the liquid-vapor coexistence curve of bulk nuclear matter since the effects of finite size, the Coulomb force, angular momentum and isospin have all been accounted for and scaled out. 


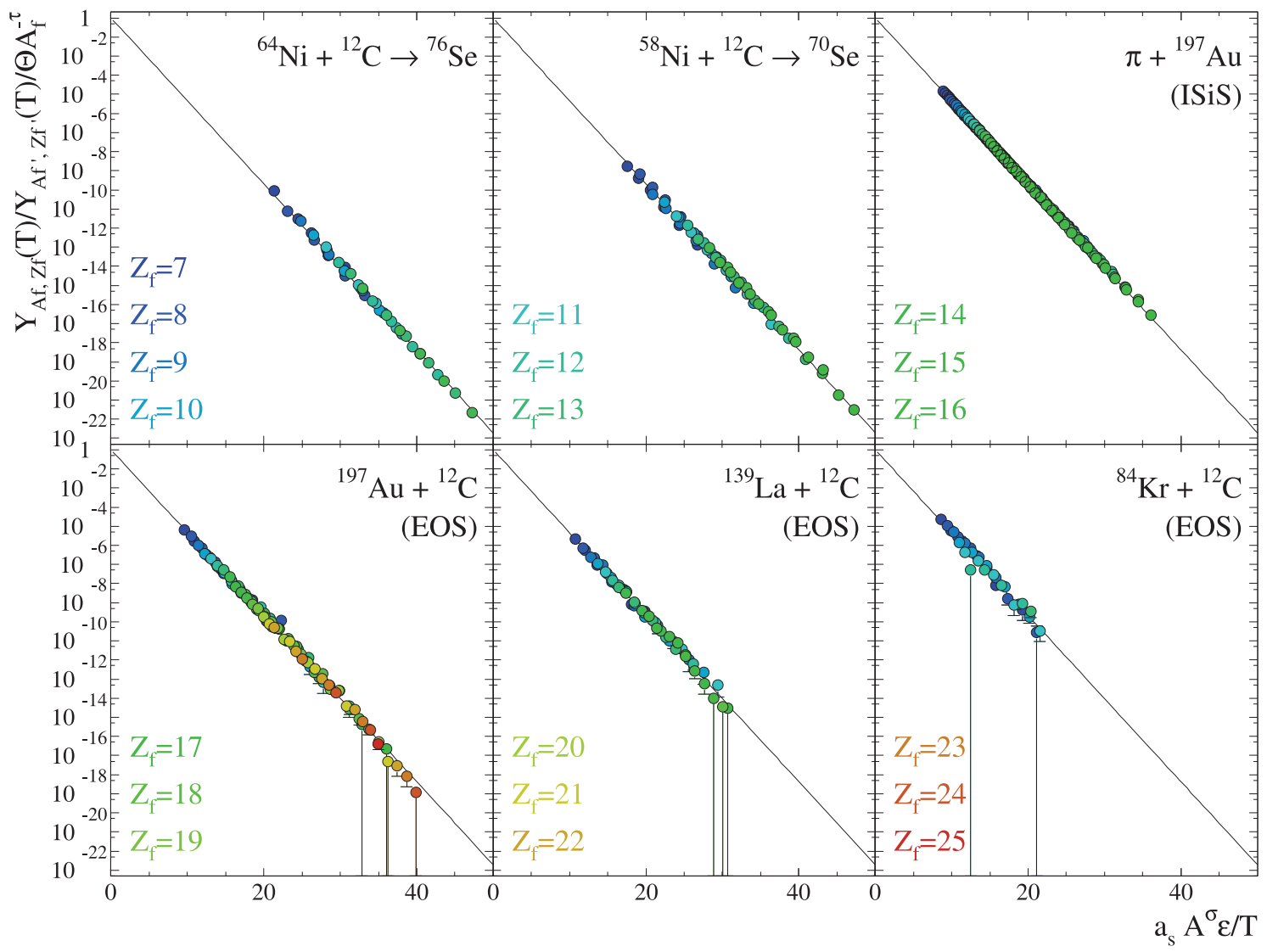

Figure 7: The scaled charged yields from the six different nuclear reactions. Over 500 points are collapsed on a single curve which describes the behavior of bulk nuclear matter. Here $\Theta$ is an effective chemical potential that takes into accounts for the effects of finite size, Coulomb force, angular momentum, and isospin dependencies. See reference [35] for further details.

\section{The nuclear phase diagrams}

With the physical cluster model and the fitting parameters from the experimental data, the nuclear phase diagrams can be created. Combining the equations from the physical cluster model and Fisher's droplet model, the pressure is written as:

$$
p=T \sum_{A} q_{0} A^{-\tau} \exp \left[-c_{0} A^{\sigma}\left(\frac{1}{T}-\frac{1}{T_{c}}\right)\right],
$$

and the critical pressure is

$$
p_{c}=T_{c} \sum_{A} q_{0} A^{-\tau}
$$

Likewise, the density is:

$$
\rho=\sum_{A} q_{0} A^{1-\tau} \exp \left[-c_{0} A^{\sigma}\left(\frac{1}{T}-\frac{1}{T_{c}}\right)\right],
$$

and the critical pressure is

$$
\rho_{c}=\sum_{A} q_{0} A^{1-\tau}
$$


First, we consider the reduced phase diagrams, where the thermodynamic variables are scaled by the critical values. Since the fits for the experimental data are done with the ratio of cluster yields, the normalization $q_{0}$ is still unknown. Considering the scaled phase diagrams removes this complication.

Also, the density of the liquid phase as a function of density is not directly measured in the experiments. The only knowledge of the liquid phase is the saturation density at zero temperature from studies of ground state nuclei.

To determine the normalization constant and to construct the liquid branch of the densitytemperature phase diagram, Guggenheim's relation of the reduced phase diagrams is used [43]:

$$
\frac{\rho_{l, v}}{\rho_{c}}=1+d_{1} \varepsilon \pm d_{\beta} \varepsilon^{\beta}
$$

In this equation, the upper sign is used for the liquid branch, the lower sign is for the vapor branch, and $\beta$ is the critical exponent $[3,4,6]$ :

$$
\beta=\frac{\tau-2}{\sigma}=0.3265 \pm 0.0001
$$

The two parameters $d_{1}$ and $d_{\beta}$ are system dependent. The reduced phase diagram for the vapor branch is then fit to these two parameters. The reduced phase diagram for the liquid branch is then generated by switching the sign as seen in equation 6.5. Furthermore the non-reduced phase diagram is created by scaling to the density of saturated nuclear matter at zero temperature. Figure 8 shows the resulting phase diagram. The critical density is found to be $\rho_{c}=0.06 \pm 0.02 \mathrm{~A} / \mathrm{fm}^{3}$.

To create the pressure-temperature phase diagram, the normalization constant found for the density is used. The resulting critical pressure is $p_{c}=0.3 \pm 0.1 \mathrm{MeV} / \mathrm{fm}^{3}$. Figure 8 also shows the pressure-temperature phase diagram.
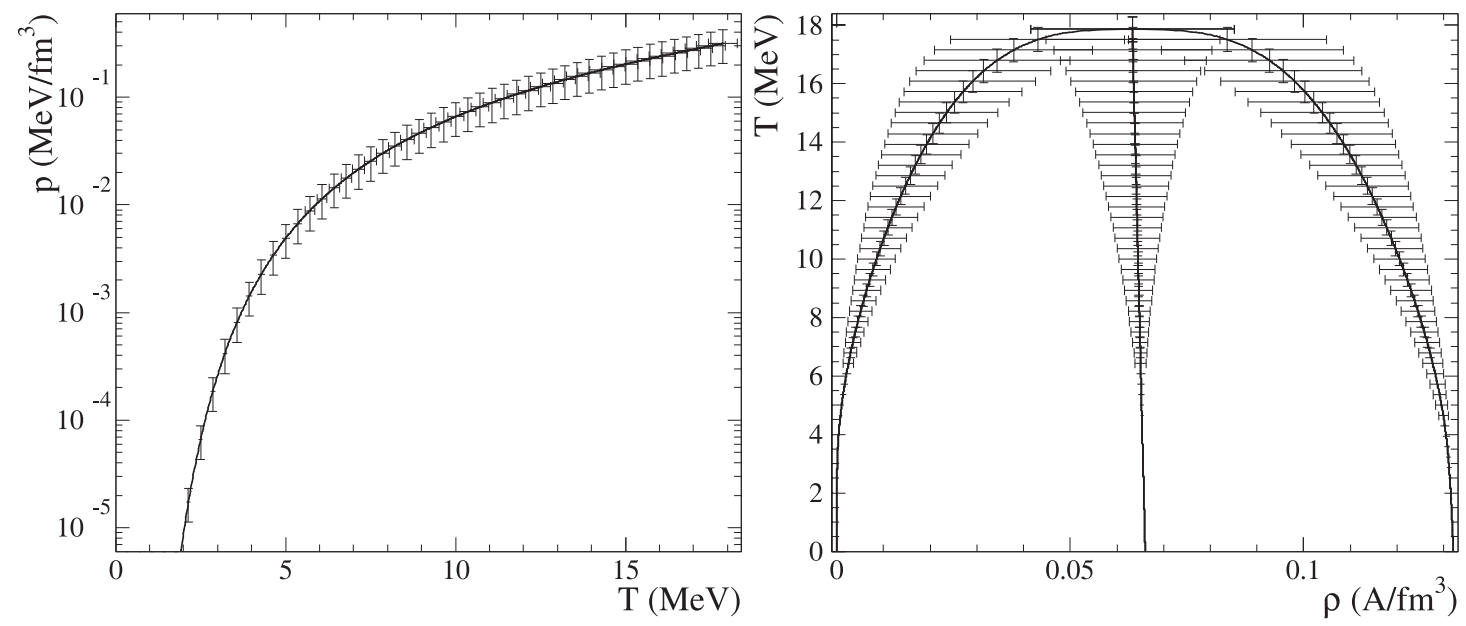

Figure 8: The extracted phase diagrams for bulk nuclear matter. Left: The pressure-temperature coexistence curve. Right: The density-temperature coexistence curve. The line in the middle represents the average density of the two phases [35]. 


\section{Conclusion}

This talk summarizes the steps taken to generate the liquid-vapor phase diagram of bulk uncharged symmetric nuclear matter. Since the typical means of measuring the thermodynamic properties of the nuclear system are not available, new techniques need to be employed.

Even though the theory of clustering in a vapor was developed independent of nuclear physics, the application to nuclear reactions is relevant. The physical cluster model in conjunction with Fisher's droplet model is shown to provide enough information to produce phase diagrams for a system.

To show that clustering in a real gas can be used to generate phase diagrams, the theory is applied to model systems. Fisher scaling is observed in both the Ising model and the LennardJones model. Furthermore, the scaling can reproduce the phase diagrams of the systems which have been determined previously through more traditional procedures.

The difference between the environment of a nuclear reaction and systems in direct coexistence also are considered. The finite size of a nucleus is shown to be taken into account through application of the complement correction. Further simulations of the Ising model and Lennard-Jones model show the validity of the complement correction.

The presence of the Coulomb repulsion in the nucleus is also discussed. Application of the transition state method reveals that the Coulomb interaction affects the decay rates by the addition of an energy barrier. Taking the extra barrier into account leaves a description of the uncharged system.

Finally, the lack of a physical vapor in contact with a decaying nuclear source is considered. The rates of evaporation from the decaying source is seen to be unchanged between a system with and without being in contact with its coexisting vapor. Furthermore, the rates of decay are directly related to the concentrations of the clusters in a coexisting system.

With a theory in place for analyzing the experimental data from nuclear reactions, the nuclear phase diagram is created. Experiments of lower energy collisions creating a compound nucleus are easily characterized, making them important in the analysis. Even though multifragmentation data is harder to characterize, the theory of cluster yields is the same and is applied in the same way.

The diverse experimental data produce consistent set of parameters for bulk nuclear matter, which allow us to create the phase diagrams of nuclear matter.

\section{References}

[1] L.G. Moretto et al., The experimental liquid-vapor phase diagram of bulk nuclear matter, J Phys G: Nucl Part Phys (2011) In Process.

[2] L. Beaulieu, L. Phair, L.G. Moretto, and G.J. Wozniak, z-dependent barriers in multifragmentation from poissonian reducibility and thermal scaling, Phys Rev Lett 81 (1998) 770.

[3] M.E. Fisher, The theory of condensation and the critical point, Physics (Long Island City, NY), 3 (1967) 255

[4] M.E. Fisher, The theory of equilibrium critical phenomena, Rep Prog Phys 30 (1967) 615.

[5] C.N. Yang and T.D. Lee, Statistical theory of equations of state and phase transitions. $i$. theory of condensation, Phys Rev 87 (1952) 404. 
Infinite symmetric nuclear matter phase diagram from low energy compound nucleus data.

Luciano Moretto

[6] T.D. Lee and C.N. Yang, Statistical theory of equations of state and phase transitions. ii. lattice gas and Ising model, Phys Rev 87 (1952) 410.

[7] R.H. Swendsen and J. Wang, Non-universal dynamics in Monte Carlo simulations, Phys Rev Lett 58 (1987) 86.

[8] A. Coniglio and W. Klein, Clusters and Ising critical droplets: a renormalisation group approach, J Phys A-Math Gen 13 (1980) 2775.

[9] A.Z. Panagiotopoulos, Direct determination of phase coexistence properties of fluids by Monte Carlo simulation in a new ensemble, Mol Phys 61 (1987) 813.

[10] T.L. Hill, Molecular clusters in imperfect gases, J Chem Phys 23 (1953) 617.

[11] C.M. Mader et al., The three-dimensional Ising model and its Fisher analysis: A paradigm of liquid-vapor coexistence in nuclear multifragmentation, Phys Rev C 68 (2003) 064601.1.

[12] P. Butera and M. Comi, Critical universality and hyperscaling revisited for Ising models of general spin using extended high-temperature series, Phys Rev B 65 (2002) 144431.1.

[13] J.P. Jeffrey and A.Z. Panagiotopoulos, Critical point and phase behavior of the pure Lennard-Jones mixture, J Chem Phys 109 (1998) 10914.1.

[14] E.W. Lemmon, M.O. McLinden, and D.G. Friend, “Thermophysical Properties of Fluid Systems” in NIST Chemistry WebBook NIST Standard Reference Database, National Institute of Standards and Technology, Gaithersburg 2005.

[15] A.L. Talapov and H.W.J. Blöte, The magnetization of the 3D Ising model, J Phys A, Math Gen 29 (1996) 5727.

[16] C.F. von Weizäcker, Zur theorie der kernmassen, Z Phys A-Hadron Nucl 96 (1937) 431.

[17] L.G. Moretto et al., The complement: A solution to liquid drop finite size effects in phase transitions, Phys Rev Lett 94 (2005) 202701.1.

[18] B. Krishnamachari, J. McLean, B. Cooper, and J. Sethna, Gibbs-Thomson formula for small island sizes: Corrections for high vapor densities, Phys Rev B 54 (1996) 8899.

[19] L.G. Moretto, J.B. Elliott, and L. Phair, Resistible effects of Coulomb interaction on nucleus-vapor phase coexistence, Phys Rev C 68 (2003) 061602(R).1.

[20] E. Wigner, The transition state method, Trans Faraday Soc 31 (1938) 29.

[21] L.G. Moretto, J.B. Elliott, and L. Phair, Compound nuclear decay and the liquid-vapor phase transition: A physical picture, Phys Rev C 72 (2005) 064605.1.

[22] T.S. Fan et al., Excitation functions and mass asymmetric fission barriers for compound nuclei ${ }^{70,76}$ Se, Nuc Phys A 679 (2000) 121.

[23] V.E. Viola et al., Light-ion-induced multifragmentation: The ISiS project, Phys Rep 434 (2006) 1.

[24] J.B. Elliott et al., Liquid to vapor phase transition in excited nuclei, Phys Rev Lett 88 (2002) 042701.1 .

[25] J.B. Elliott et al., Constructing the phase diagram of finite neutral nuclear matter, Phys Rev C 67 (2003) 024609.1.

[26] M.L. Gilkes et al., Determination of critical exponents from the multifragmentation of gold nuclei, Phys Rev Lett 73 (1994) 1590. 
Infinite symmetric nuclear matter phase diagram from low energy compound nucleus data.

Luciano Moretto

[27] K. Kwiatkowski et al., The Indiana silicon sphere $4 \pi$ charged-particle detector array, Nucl Instrum Methods 360 (1995) 571.

[28] J.A. Hauger et al., Dynamics of the multifragmentation of 1 AGeV gold on carbon, Phys Rev Lett 77 (1996) 235.

[29] T. Lefort et al., Heating ${ }^{197}$ Au nuclei with 8 GeV/c antiproton and $\pi^{-}$beams, Phys Rev Lett 83 (1999) 4033.

[30] J.A. Hauger et al., Two-stage multifragmentation of 1 AGeV Kr, La, and Au, Phys Rev C 62 (2000) 024616.1 .

[31] L. Beaulieu et al., Signals for a transition from surface to bulk emission in thermal multifragmentation, Phys Rev Lett 84 (2000) 5971.

[32] J.B. Elliott et al., Nuclear multifragmentation, percolation, and the Fisher droplet model: Common features of reducibility and thermal scaling, Phys Rev Lett 85 (2000) 1194.

[33] J.B. Elliott et al., Statistical signatures of critical behavior in small systems, Phys Rev C 62 (2000) 064603.1 .

[34] A.S. Hirsch et al., Experimental results from high energy proton-nucleus interactions, critical phenomena, and the thermal liquid drop model of fragment production, Phys Rev C 29 (1984) 508.

[35] J.B. Elliott, L.G. Moretto, L. Phair, and P.T. Lake, Experimentally determining the liquid-vapor coexistence curve of bulk nuclear matter, Phys Rev C (2011) In Process.

[36] K. Hagel et al., Production and characterization of hot nuclei in the reactions of 19 and $35 \mathrm{MeV} / \mathrm{u}^{14} \mathrm{~N}$ and ${ }^{145}$ Sm, Nucl Phys A 486 (1988) 429.

[37] Al.H. Raduta and Ad.R. Raduta, Simulation of statistical ensembles suitable for the description of multifragmentation, Phys Rev C 55 (1997) 1344.

[38] P. Chomaz, M. Colonna, and J. Randrup, Nuclear spinodal fragmentation, Phys Rep 389 (2004) 263.

[39] B. Friedman and V.R. Pandharipande, Hot and cold, nuclear and neutron matter, Nucl Phys A 361 (1981) 502 .

[40] N.K. Glendenning, L.P. Csernai, and J.I. Kapusta, Liquid-gas phase separation in nuclear collisions, Phys Rev C 33 (1986) 1299.

[41] H. Müller and B.D. Serot, Phase transitions in warm, asymmetric nuclear matter, Phys Rev C 52 (1995) 2072.

[42] M. Jin, M. Urban, and P. Schuck, BEC-BCS crossover and the liquid-gas phase transition in hot and dense nuclear matter, Phys Rev C 82 (2010) 024911.1.

[43] E.A. Guggenheim, The principle of corresponding states, J Chem Phys 13 (1945) 253. 